Check for updates

Cite this: J. Mater. Chem. A, 2021, 9, 14731

Received 27th April 2021

Accepted 11th June 2021

DOI: $10.1039 / \mathrm{d} 1 \mathrm{ta0} 3543 f$

rsc.li/materials-a

\title{
Hybrid water vapor sorbent design with pollution shielding properties: extracting clean water from polluted bulk water sources $\uparrow$
}

\author{
Renyuan Li, (D) a Mengchun Wu, (D) a Yusuf Shi, ${ }^{a}$ Sara Aleid, ${ }^{a}$ Wenbin Wang, (D) a \\ Chenlin Zhang ${ }^{a}$ and Peng Wang iD *ab
}

\begin{abstract}
The shortage and contamination of local water resources have long been a challenge especially for off-grid communities without centralized water supply. The emerging solar photothermal distillation lacks the capability of handling polluted source water with a wide range of common environmental pollutants. Based on water vapor harvesting, this work reports a Simple Water Extraction Apparatus with Pollutant Shielding (SWEAPS) design which is able to efficiently produce clean water from various polluted liquid water sources and the atmosphere. SWEAPS is fabricated by encapsulating a water vapor sorbent by an omniphobic fabric. The omniphobicity of the encapsulation fabric endows SWEAPS with self-floating capability and the ability to screen out the contaminants in the source water. The self-floating properties of SWEAPS allow it to harvest clean water vapor right above the source-water-air interface where the relative humidity is close to $100 \%$, leading to its much higher water harvesting capacity than that of the same material harvesting water vapor from the ambient atmosphere. Due to the chemical and physical stability, anti-bacterial, pollution and corrosion shielding effects of SWEAPS, it is demonstrated to produce clean water meeting the WHO drinking water standard from various polluted water resources, such as seawater, contaminated water, and amazingly untreated real domestic wastewater. SWEAPS has the potential to produce clean water for point of consumption at a decentralized scale and thus to improve the quality of life for those who need water most.
\end{abstract}

\section{Introduction}

In recent years, to alleviate the shortage of freshwater, the distributed freshwater production approaches that are powered by low-grade energy, especially solar photothermal distillation, have attracted considerable attention and demonstrated their potential in producing clean water for point of consumption. ${ }^{\mathbf{1 - 4}}$ $\sim 144$ million people globally still rely on untreated surface water as the water source, which is often contaminated chemically, physically and/or biologically. ${ }^{5-7}$ Under such conditions, photothermal distillation, because of its in situ heating, accelerates the release of volatile pollutants from the source water and their subsequent collection in the produced water. ${ }^{8}$ Moreover, due to the direct contact between the photothermal materials and the bulk source water, the photothermal materials are prone to contamination and fouling by pollutant

${ }^{a}$ Water Desalination and Reuse Center, Division of Biological and Environmental Science and Engineering, King Abdullah University of Science and Technology, Thuwal 23955-6900, Saudi Arabia. E-mail: peng.wang@kaust.edu.sa; peng1.wang@ polyu.edu.hk

${ }^{b}$ Hong Kong Polytechnic University, Hung Hom, Kowloon, Hong Kong, China

$\dagger$ Electronic supplementary information (ESI) available. See DOI: $10.1039 / \mathrm{d} 1 \mathrm{ta} 03543 \mathrm{f}$ species in the source water, such as dissolved salts, natural organic matter, and bacteria. ${ }^{9-12}$

On the other hand, sorption-based atmospheric water harvesting $(\mathrm{AWH})$ is being intensively studied for collecting clean water from the atmosphere even under dry conditions. ${ }^{13-19}$ In a sense, the AWH process can be considered as harvesting the relatively clean "pre-distilled" water vapor from the ambient air and direct contact between the vapor sorbent and the source bulk water is avoided..$^{20}$ Moreover, the vapor sorption takes place at the ambient temperature instead of the elevated temperature as in photothermal distillation. ${ }^{21-24}$ Thus, the AWH based approach is more advantageous over the photothermal distillation in terms of the quality of the produced water. However, the clean water production rate of the $\mathrm{AWH}$ based approach is relatively low especially at dry areas due to the low humidity therein. ${ }^{25-28}$

This work, for the first time, reported a simple but versatile strategy that can produce clean water from contaminated bulk source water via an AWH design that shields the contaminating species in the bulk water. A Simple Water Extraction Apparatus with Pollutant Shielding (SWEAPS) was devised by encapsulating an AWH sorbent by an omniphobic fabric layer. Owing to the omniphobicity of the fabric, SWEAPS can self-float on the surface of the regular bulk source water, where the RH is close 
to $100 \%$ to ensure a high water uptake rate. The design of SWEAPS physically separates the source bulk water from the vapor sorbent, giving rise to its expected pollutant shielding properties. Due to the high selectivity of the sorbent towards water vapor, the volatile compounds in the source water are much less a concern for SWEAPS than regular solar photothermal distillation.

In this work, the performance of SWEAPS was evaluated with multiple types of quality-impaired source water, including mud, synthetic water contaminated with an acid, base, dye, and heavy metal, seawater, and real untreated domestic wastewater. With SWEAPS floating on top of the bulk source water, a much higher water uptake rate (i.e., $\sim 1.57 \mathrm{~g} \mathrm{~g}^{-1}$ ) was obtained than that of the same sorbent harvesting water vapor from the ambient air (i.e., $\sim 0.81 \mathrm{~g} \mathrm{~g}^{-1}$ ). SWEAPS was demonstrated to be stable, noncorrosive to metal, and to effectively shield the pollutants in the contaminated source water from reaching the produced water. The produced water, even the one produced from the untreated domestic wastewater, had the measured contaminants' concentrations all below the WHO drinking water standards. This work widens our approaches for producing freshwater from unconventional water sources and thus is an important contribution to global water sustainability.

\section{Materials and methods}

\section{Chemicals and materials}

Acrylamide (AM, 99\%), $N, N^{\prime}$-methylenebis(acrylamide) (MBAA, $99 \%$ ), $N, N, N^{\prime}, N^{\prime}$-tetramethylethylenediamine (TEMED, 99\%), tetraethylorthosilicate (TEOS, 99\%), branched polyethylenimine (bPEI, $\left.M_{\mathrm{w}} \sim 25000\right), 1 H, 1 H, 2 H, 2 H$-perfluorooctyltriethoxysilane (POTS, 99\%), 1H,1H,2H,2H-perfluorodecanethiol (PFDT, 99\%), polyvinylpyrrolidone (PVP, $\left.M_{\mathrm{w}} \sim 40000\right)$, sodium citrate tribasic dihydrate (Cit-Na, 99\%), hydrogen peroxide (30 wt\%), sodium borohydride $\left(\mathrm{NaBH}_{4}, 99 \%\right)$, silver nitrate $\left(\mathrm{AgNO}_{3}, 99.8 \%\right)$, and calcium chloride $\left(\mathrm{CaCl}_{2}, 99 \%\right)$ were purchased from SigmaAldrich. Cotton fabric was purchased from Fisher Scientific. Methanol and 2-propanol were purchased from VWR. Ammonium hydroxide (29 wt\%) was purchased from Fisher Chemical. Potassium persulfate (KPS, 99\%) was purchased from Acros Organics. Deionized water (DI water, 18.2 M , from a Milli-Q system) was used throughout the synthesis process.

\section{Synthesis of PAM- $\mathrm{CaCl}_{2}$ hydrogel}

The synthesis process of $\mathrm{PAM}-\mathrm{CaCl}_{2}$ was in accord with our previous work. ${ }^{20}$ Briefly, $24 \mathrm{~g}$ of AM was dissolved in $120 \mathrm{~mL}$ of DI water, followed by purging with nitrogen gas to eliminate the dissolved oxygen. Then $0.01 \mathrm{~g}$ of MBAA and $0.12 \mathrm{~g}$ of KPS were added to the AM solution as a cross-linking agent and initiator, respectively. Finally, $100 \mu \mathrm{L}$ of TEMED was added as the crosslinking accelerator. The PAM hydrogel was obtained after 6 hours of reaction at room temperature. The as-prepared PAM hydrogel was then freeze-dried at $-80{ }^{\circ} \mathrm{C}$ (FreeZone 2.5 plus, LABCONCO) and then immersed in $120 \mathrm{~mL}$ of $\mathrm{CaCl}_{2}$ solution $\left(0.4 \mathrm{~g} \mathrm{~mL}^{-1}\right)$ for 3 days under ambient conditions to fabricate the $\mathrm{PAM}-\mathrm{CaCl}_{2}$ hydrogel.

\section{Preparation of the omniphobic fabric}

First, silver nanoparticles (AgNPs) were synthesized following a method reported in the literature. ${ }^{29}$ Briefly, $202.5 \mathrm{mg}$ of $\mathrm{AgNO}_{3}$ was dissolved in $1455 \mathrm{~mL}$ of DI water, followed by adding $55.5 \mathrm{mg}$ of PVP and $5.55 \mathrm{~g}$ of Cit-Na. Then $28.8 \mathrm{~mL}$ of $\mathrm{H}_{2} \mathrm{O}_{2}$ was dropped into the mixed solution and vigorously stirred for 1 hour at room temperature. Finally, $78 \mathrm{~mL}$ of $100 \mathrm{mM} \mathrm{NaBH}_{4}$ solution was slowly added to the mixed solution under magnetic stirring. A AgNP dispersion was obtained after 12 hours of reaction at room temperature.

A piece of $20 \times 20 \mathrm{~cm}^{2}$ cotton fabric was immersed in a mixture of $60 \mathrm{~mL}$ methanol, $180 \mathrm{~mL}$ of 2-propanol, and $45 \mathrm{~mL}$ of ammonium hydroxide. Then $6.4 \mathrm{~mL}$ of TEOS was dropped into the mixture and then reacted overnight at room temperature under mechanical shaking to obtain the $\mathrm{SiO}_{2}$-coated cotton fabric ( $\mathrm{SiO}_{2} @$ cotton). The $\mathrm{SiO}_{2} @$ @otton was washed with methanol for 5 min 3 times under ultrasonic conditions before drying at $60{ }^{\circ} \mathrm{C}$ for $1 \mathrm{~h}$. Then the as-prepared $\mathrm{SiO}_{2}$ @cotton was immersed in $1.0 \mathrm{~L}$ of $1.0 \mathrm{mg} \mathrm{mL} \mathrm{m}^{-1} \mathrm{~b}$-PEI solution for $30 \mathrm{~min}$, followed by thoroughly washing with DI water. The b-PEI modified fabric was subsequently immersed in a mixture of $200 \mathrm{~mL}$ of AgNP dispersion and $200 \mathrm{~mL}$ DI water under mechanical stirring overnight to obtain the AgNP coated fabric (AgNPs@SiO ${ }_{2} @$ cotton). The AgNPs@SiO ${ }_{2} @$ cotton was then washed with DI water and dried at $60{ }^{\circ} \mathrm{C}$. Finally, the omniphobic F-AgNPs@SiO ${ }_{2} @$ cotton fabric was obtained by using 50 $\mu \mathrm{L}$ of POTS and PFDT to modify the AgNPs@SiO ${ }_{2}$ @cotton through a chemical vapor deposition (CVD) method at $80^{\circ} \mathrm{C}$ for 8 hours.

\section{Water uptake from different source water}

Characterization and details of the DVS test can be found in ESI Note $1 . \dagger$ The water uptake of SWEAPS was conducted with 9 different water resources: ambient air, DI water, water from the Red Sea, acidic solution ( $\mathrm{pH}=1 \mathrm{HCl}$ ), basic solution $(\mathrm{pH}=13$ $\mathrm{NaOH}$ ), mud (from an artificial pond in KAUST), dye solution (20 ppm rhodamine B), heavy metal (100 ppm $\mathrm{CuSO}_{4}$ ) contaminated water, and domestic wastewater influent (from the KAUST wastewater treatment plant and pre-filtered to remove solid components). For the water uptake test from the ambient air, $1.5 \mathrm{~g}$ of pre-dried $\mathrm{PAM}-\mathrm{CaCl}_{2}$ hydrogel was encapsulated in a $\sim 4.5 \times 9 \times 0.8 \mathrm{~cm}^{3}$ omniphobic FAgNPs@SiO ${ }_{2} @$ cotton fabric and thus exposed to the ambient air. The weight change of SWEAPS was recorded using a computer-connected electronic balance. $1.5 \mathrm{~g}$ of pre-dried PAM-CaCl ${ }_{2}$ hydrogel without omniphobic fabric was used for comparison.

For water uptake tests from the bulk-phase water, SWEAPS with $2.0 \mathrm{~g}$ of pre-dried PAM- $\mathrm{CaCl}_{2}$ hydrogel was floated on the surface of the source water. The weight changes of SWEAPS were manually recorded every $30 \mathrm{~min}$. The total duration of the test was 8 hours. The RH at the surface of each source water was measured prior to the test. The humidity sensor was placed $\sim 1 \mathrm{~cm}$ above the water surface. The sorbent regeneration and water collection processes were conducted in a condensation chamber via a photothermal approach. To assist evaporation, 
a commercial spectrally selective absorber (SSA) layer was used to cover the top side of the saturated SWEAPS. The metal chamber was capped with a transparent dish as the light path and a solar simulator (92023A, Newport) was used to irradiate the materials. The room temperature and the relative humidity $(\mathrm{RH})$ of the lab were controlled by a heating, ventilation and airconditioning (HVAC) system. The room temperature was maintained at $\sim 22{ }^{\circ} \mathrm{C}$ and the $\mathrm{RH}$ of the lab was maintained at $\sim 60 \%$.

\section{Stability cycling test}

The cycling test was performed to identify the stability of the device during the water uptake and water release process. The weight of the omniphobic fabric and the pre-dried PAM- $\mathrm{CaCl}_{2}$ hydrogel in the test was $0.47 \mathrm{~g}$ and $2.06 \mathrm{~g}$, respectively. The test consisted of 10 cycles, with each cycle including an 8 hour water uptake process from bulk DI water and a 3 hour photothermalassisted water release process under simulated sunlight $(1 \mathrm{~kW}$ $\mathrm{m}^{-2}$ ). The weight change of the SWEAPS was measured in each individual test and the contact angle of water on the omniphobic fabric was measured before and after the cycling test.

\section{Outdoor tests}

Outdoor field tests were performed based on water uptake from the Red Sea and atmosphere. Both tests were conducted with SWEAPS that contained $15 \mathrm{~g}$ of pre-dried PAM-CaCl $\mathrm{C}_{2}$ hydrogel.

For the AWH outdoor test, SWEAPS was placed on an electronic balance to record the weight change. The water uptake process was started at 18:00, Nov. 17, 2020, and ended at 6:00 am on Nov. 18, 2020. Then, the saturated SWEAPS was transferred into a $20 \times 20 \times 5 \mathrm{~cm}^{3}$ metal chamber for the water desorption and collection test. The top of the metal chamber was covered with a polypropylene film as a transparent window. A commercial spectrally selective absorber (SSA) was placed inside the chamber as the photothermal component to obtain a high temperature and heat SWEAPS. The metal chamber was tilted $\sim 20^{\circ}$ facing the south to ensure a stronger solar irradiance, and the test was performed between 14:30 and 16:30, Nov. $18,2020$.

For the Red Sea test, SWEAPS was chained on a mangrove branch, and floated on the surface of Red Sea water in the KAUST campus for 8 hours $(16: 00-24: 00$, Dec. 17, 2020) for the water uptake process. Then, the water desorption and collection process was conducted with a battery-powered electronic heater for 1 hour and $30 \mathrm{~min}$ (1:00-2:30 am, Dec. 18, 2020).

\section{Results and discussion}

\section{Fabrication of SWEAPS}

Modelling (ESI Note $2 \dagger$ ) was performed first to theoretically investigate the humidity distribution above the bulk water surface under practical outdoor conditions. The modeling results indicate that, with the presence of wind flow across the water surface, the high humidity region with $\mathrm{RH}$ of $\sim 100 \%$ only occurs within a short range above the water surface (i.e., less than 5-10 cm). The humidity profile quickly declined to the value of ambient humidity. Therefore, it is important to ensure close contact between the AWH-based water collection device and water surface to gain a superior water sorption rate. Mass transfer calculation indicates that, in comparison with adsorbing water vapor from ambient air, the mass transfer rate of the water vapor from the bulk water to the sorbent placed within the high humidity region above the bulk water surface is significantly faster.
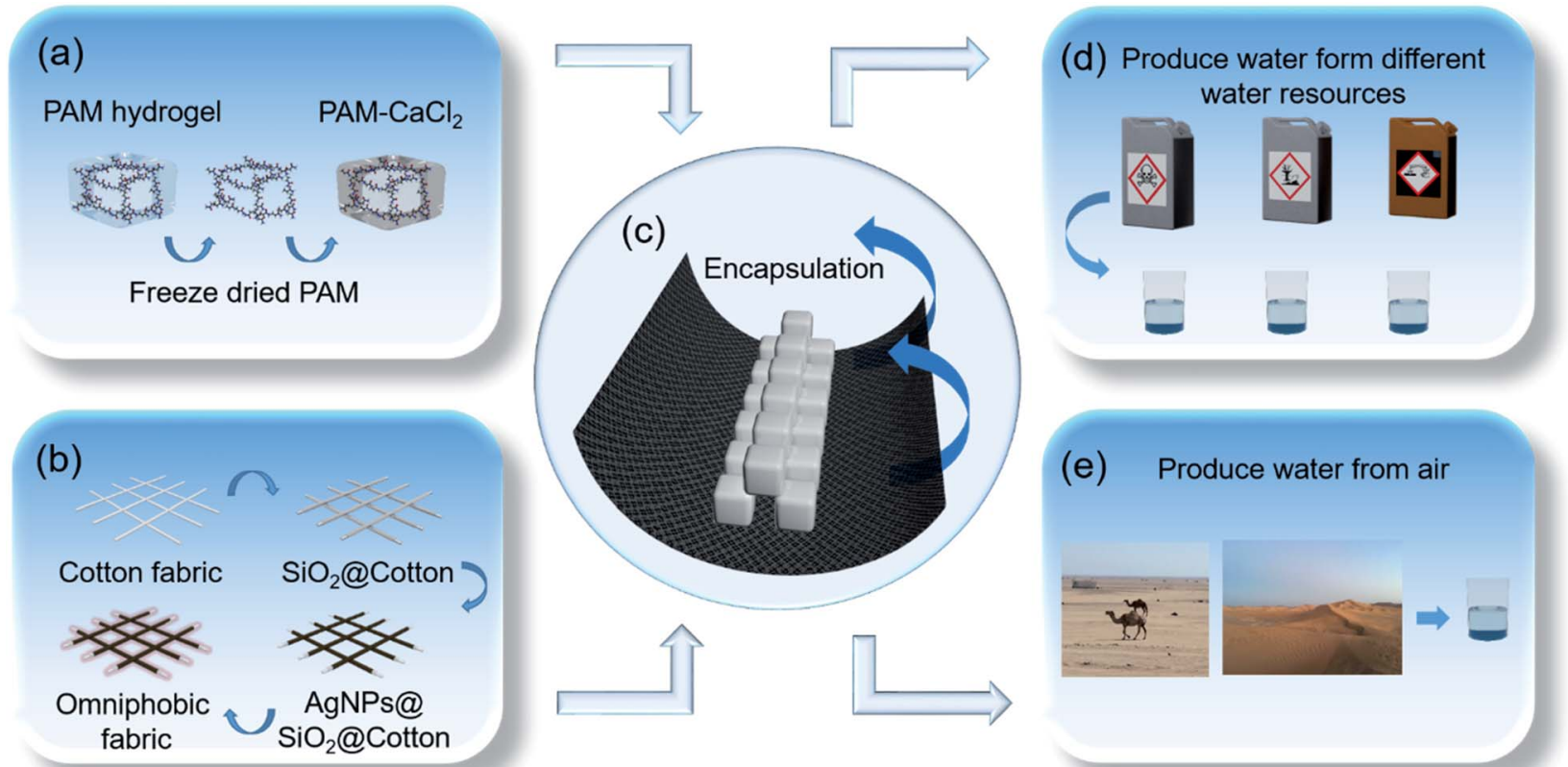

Fig. 1 Schematic illustrations of the fabrication process of (a) PAM- $\mathrm{CaCl}_{2}$ hydrogel, (b) omniphobic encapsulation layer, and (c) SWEAPS. Applications of SWEAPS for (d) water production from wide ranges of bulk water types and (e) atmospheric water harvesting. 
SWEAPS consists of a well-studied AWH material encapsulated inside a porous fabric omniphobic container. Polyacrylamide-calcium chloride ( $\left.\mathrm{PAM}-\mathrm{CaCl}_{2}\right)$ sorbent was chosen as the AWH component (Fig. 1a) and cotton fabric was used as the matrix to fabricate an omniphobic layer. The fabric was firstly modified with silica $\left(\mathrm{SiO}_{2}\right)$ and silver nanoparticles (AgNPs) followed by grafting perfluorosilane and perfluorodecane to enable its omniphobicity (Fig. 1b). The PAM$\mathrm{CaCl}_{2}$ sorbent was finally encapsulated into the omniphobic fabric container (Fig. 1c) to deliver the final SWEAPS water sorption system, which is expected to effectively produce clean water from various polluted liquid-water resources (Fig. 1d) as well as from ambient air (Fig. 1e).

The $\mathrm{PAM}-\mathrm{CaCl}_{2}$ hydrogel was used as the water vapor sorbent in this design due to its low cost, high water uptake capacity, stability, and simple synthesis process. ${ }^{\mathbf{1 6 2 0}}$ The PAM substrate was prepared via in situ polymerization of AM monomers in the aqueous phase. After freeze-drying, the resultant porous PAM was immersed in the $\mathrm{CaCl}_{2}$ aqueous solution to obtain the hybrid hydrogel (PAM- $\left.\mathrm{CaCl}_{2}\right)$. The PAM$\mathrm{CaCl}_{2}$ was then cut into small cubes to enhance the vapor sorption/desorption kinetics. The omniphobic encapsulation fabric layer was fabricated based on the three-stage modification procedure (Fig. 1b). The cotton fabric was chosen to fabricate the omniphobic encapsulation layer due to its low cost and porous structure (Fig. 2a). Since the surface of the cotton fibers was smooth (Fig. 2b), $\mathrm{SiO}_{2}$ and AgNPs were applied to improve the roughness of the cotton fibers. The cotton fabric was first coated with a layer of $\mathrm{SiO}_{2}$ ( $\mathrm{SiO}_{2}$ @cotton) by the traditional Stöber method (Fig. 2c and d) and the $\mathrm{SiO}_{2}$ also provides reaction sites to anchor perfluorosilane with $-\mathrm{Si}\left(\mathrm{OCH}_{3}\right)_{3}$ end groups for the final modification. ${ }^{30}$ A layer of AgNPs was coated on the surface of $\mathrm{SiO}_{2}$ @cotton to further increase the surface roughness (Fig. 2e-g) and dye the $\mathrm{SiO}_{2}$ @cotton fabric into a dark appearance due to the inhomogeneous size and shape of AgNPs. In order to modify AgNPs onto the surface of $\mathrm{SiO}_{2}$ @cotton, positively charged b-PEI was first coated on the surface of $\mathrm{SiO}_{2}$ @cotton through electrostatic interactions, and the negatively charged AgNPs were subsequently deposited onto the surface of b-PEI coated $\mathrm{SiO}_{2} @$ cotton through electrostatic interactions. AgNPs can not only improve the surface roughness and photothermal properties, but also endow the cotton fabric with anti-bacterial properties. ${ }^{29,31-33}$

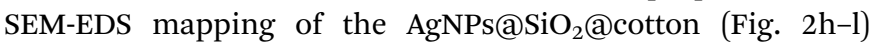
shows the homogeneous elemental distribution of $\mathrm{Si}$ and $\mathrm{Ag}$, confirming the successful modification of the cotton fabric.

$1 H, 1 H, 2 H, 2 H$-perfluorooctyltriethoxysilane (POTS) and $1 H, 1 H, 2 H, 2 H$-perfluorodecanethiol (PFDT) were applied to modify AgNPs@SiO ${ }_{2} @$ cotton via a chemical vapor deposition (CVD) method to endow the fabric with omniphobic performance (F-AgNPs@SiO ${ }_{2} @$ cotton). The silane termination of POTS enables a covalent linkage between the silica and POTS chain, while the thiol termination of PFDT secures its covalent linkage with AgNPs. The XPS spectrum shows that, after the CVD process, a strong $\mathrm{F}$ 1s peak, corresponding to $46.7 \%$ of surface chemical composition, was observed (Fig. 3a), indicating the successful deposition of the perfluoro-components. XPS analysis further substantiates the chemical composition of the fabricated F-AgNPs@SiO ${ }_{2} @$ cotton (Fig. 3a, ESI Note 3, Fig. S1-S4†).

The surface wetting behavior of F-AgNPs@SiO ${ }_{2} @$ cotton was evaluated by using eight different liquids, including acetone, silicon oil, octane, butanol, toluene, ethyl acetate (EA), dimethyl sulfoxide (DMSO), and water. The corresponding contact angle (CA) was $106.3^{\circ}, 129.8^{\circ}, 132.2^{\circ}, 139.1^{\circ}, 140.5^{\circ}, 145.7^{\circ}, 152.7^{\circ}$, and $153.2^{\circ}$, respectively (Fig. $3 \mathrm{~b}$ ), indicating that F-
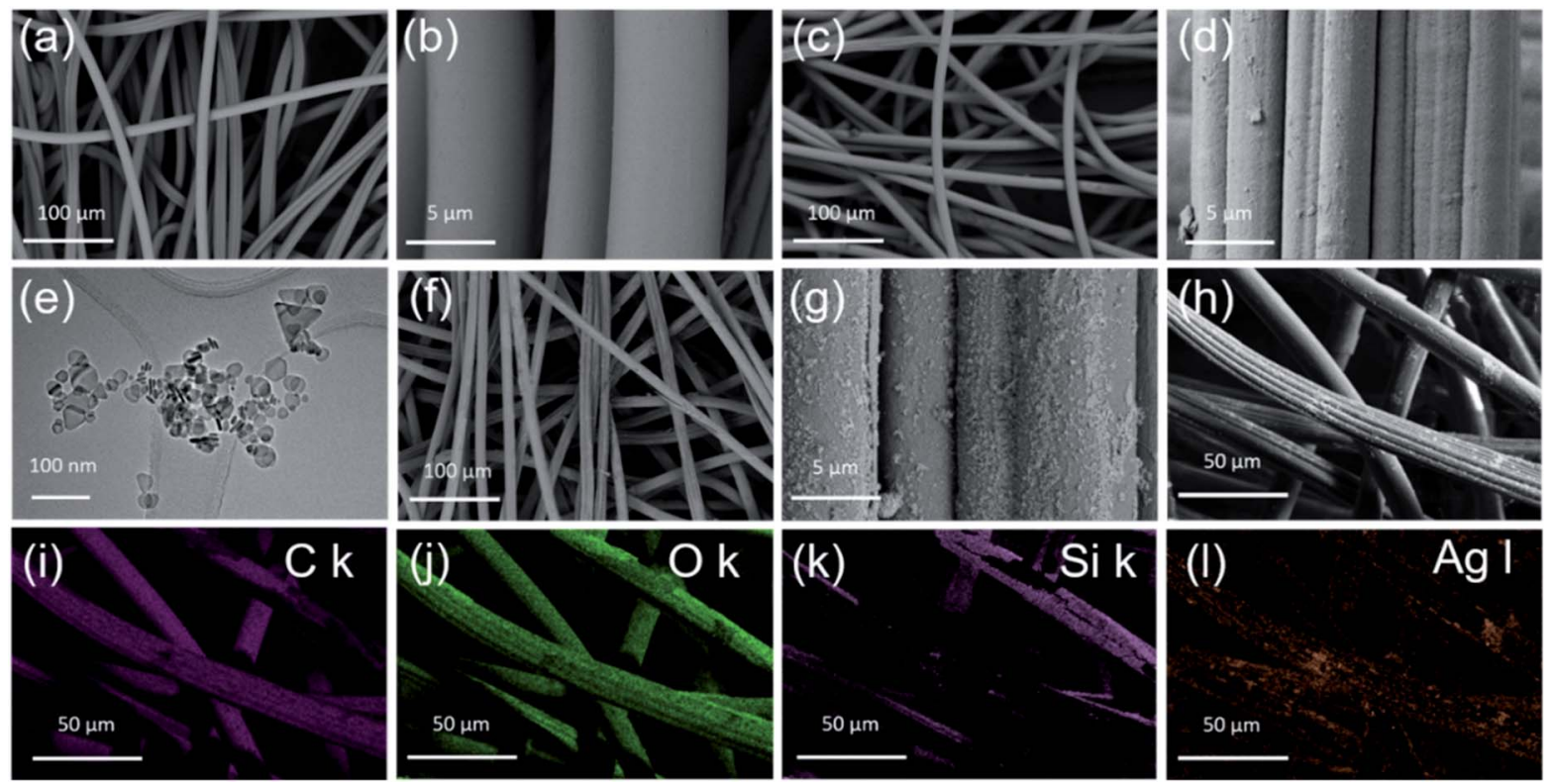

Fig. 2 SEM images of ( $a$ and b) the cotton fabric and (c and d) $\mathrm{SiO}_{2}$ coated cotton fabric. (e) HRTEM image of AgNPs. SEM images of ( $f$ and g) AgNP coated $\mathrm{SiO}_{2}$ @ cotton. (h-l) SEM-EDS mapping of AgNPs@SiO ${ }_{2}$ acotton. 
(a)

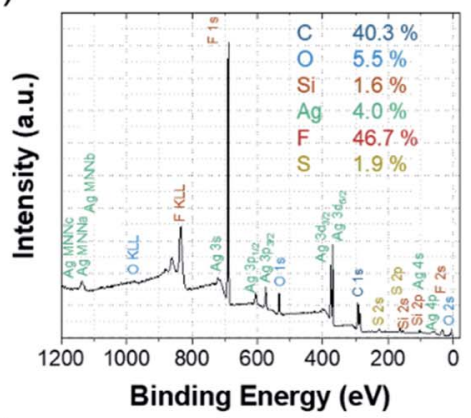

(c)

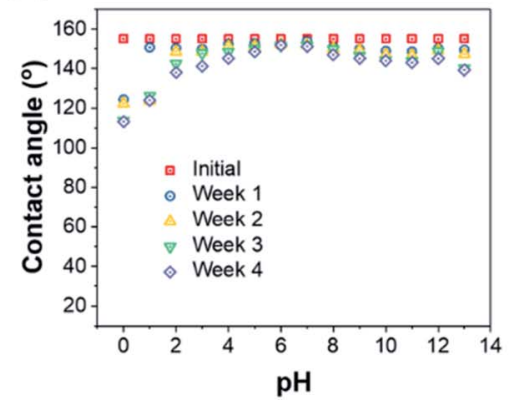

(b)

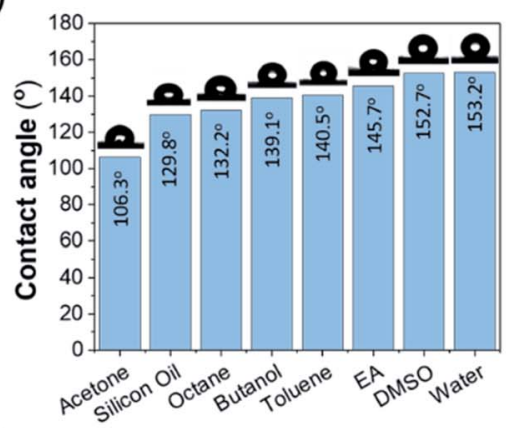

(d) $p$

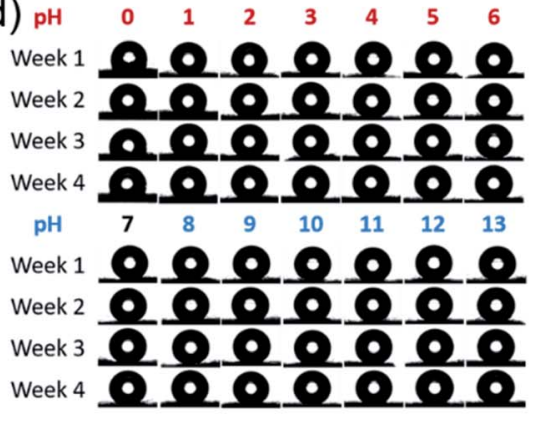

Fig. 3 (a) XPS spectrum of F-AgNPsaSiO ${ }_{2}$ acotton. (b) The CA of F-AgNPs@SiO ${ }_{2}$ acotton fabric with different liquids. (c) Time-dependent CA change of $\mathrm{F}-\mathrm{AgNPs}\left(\mathrm{SiO}_{2}\right.$ (acotton in the solution with different $\mathrm{pH}$. (d) $\mathrm{CA}$ images of water droplets with different $\mathrm{pH}$ on the surface of $\mathrm{F}$ AgNPsCSiO 2 acotton.

AgNPs@SiO $\mathrm{O}_{2} @$ cotton has water- and oil-repellent properties, namely, omniphobicity. Due to its omniphobicity and light weight, SWEAPS can self-float on the surface of various polluted source waters (more details are presented in the "water uptake from different water sources" section).

The durability and stability of F-AgNPs@SiO $\mathrm{S}_{2} @$ cotton were investigated by using acidic or alkali solutions. The tests were carried out by floating F-AgNPs@SiO $\mathrm{Si}_{2} @$ cotton with an initial water CA of $\sim 154^{\circ}$ on the surface of the solutions with the $\mathrm{pH}$ ranging from 0 to 13 and the testing solutions were then covered to minimize water evaporation. As shown in Fig. 3c and d, after a one-month test, F-AgNPs@SiO ${ }_{2}$ acotton still showed excellent hydrophobicity in all cases. Its water CA dropped a bit to $113.1^{\circ}$ and $123.9^{\circ}$ with the highly corrosive $\mathrm{pH}=0$ and $\mathrm{pH}=1$ aqueous solutions while the CA change was insignificant with solutions with a $\mathrm{pH}$ range of $2-13$, indicating the long-term stability of the omniphobic F-AgNPs@SiO ${ }_{2} @$ cotton under extreme conditions. Besides, the wetting behavior of F-AgNPs@SiO ${ }_{2} @$ cotton was tested in a $15 \% \mathrm{v} / \mathrm{v}$ waste pump oil-water mixture. A piece of $\mathrm{F}$ AgNPs@SiO ${ }_{2} @$ cotton was floated on the surface of the oil-water mixture for 48 hours and no wetting was observed (Fig. S5†), showing its stable oil repellent properties.

Most of the water vapor sorbents contain halide ions that show a strong corrosion tendency to metals. ${ }^{34,35}$ Meanwhile, the leakage of the liquid-state sorbent composition may result in the degradation of sorbent properties. Therefore, corrosion shielding and liquid repellent properties are highly desired for improving the reliability of the vapor sorbents and expanding the application scope of the sorbents. The corrosion shielding tests were conducted by putting the $\mathrm{PAM}-\mathrm{CaCl}_{2}$ hydrogels with and without the omniphobic AgNPs@SiO ${ }_{2} @$ cotton fabric layer on the copper plates (Fig. S6 $\dagger$ ). As can be seen, the copper plate showed obvious corrosion 2 months after coming into contact with the pristine $\mathrm{PAM}-\mathrm{CaCl}_{2}$ hydrogel under room conditions, and the $\mathrm{PAM}-\mathrm{CaCl}_{2}$ hydrogel turned blue due to the migration of the copper ions from the corroded copper plate (Fig. S6a $\dagger$ ). In comparison, no corrosion was observed on the copper plate in the case with the omniphobic fabric layer, and the hydrogel retained its original white color (Fig. S6b†). This is because the omniphobic fabric layer can prevent direct contact between the metal surface and the $\mathrm{CaCl}_{2}$ liquid, thus preventing the corrosion of the metal. The above results confirm the corrosion shielding and liquid repellent properties of the omniphobic fabric, which allows SWEAPS to be directly placed on top of a SSA plate and also permits direct heating of SWEAPS using metal electric heating plates without the risk of metal surface corrosion. The corrosion shielding performance of the omniphobic fabric, along with its porous and liquid-repellent ability, makes it a general protective approach to address the corrosion issue in a wide variety of potential application scenarios.

\section{Evaluation of water uptake performance}

Since the working principle of SWEAPS is based on the AWH process, a dynamic water vapor sorption (DVS) test was conducted to evaluate the water sorption properties of the AWH material at different RHs (Fig. 4a). As expected, the water sorption rate is significantly increased with increasing the RH. On the other hand, the real-time water uptake capacity during 
(a)

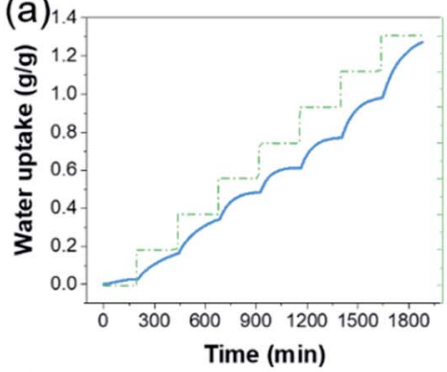

(d)

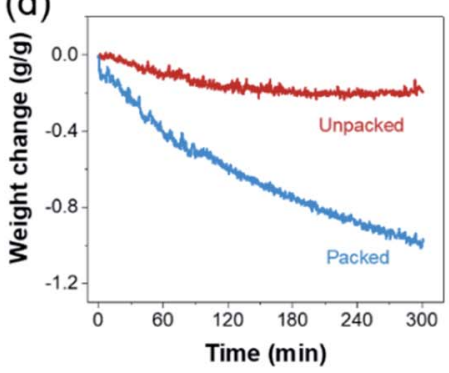

(g)

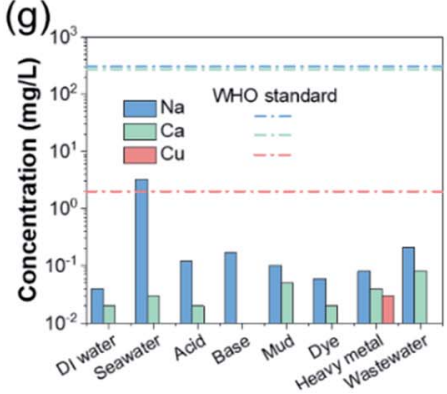

(b)

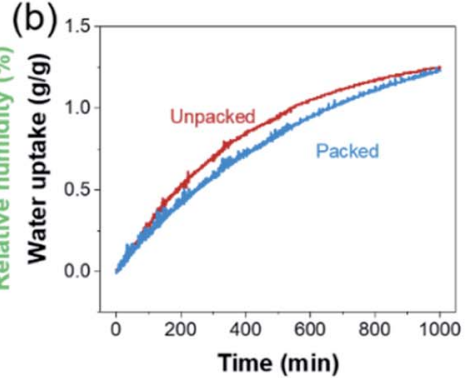

(e)

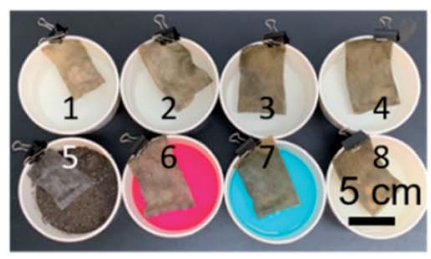

(h)

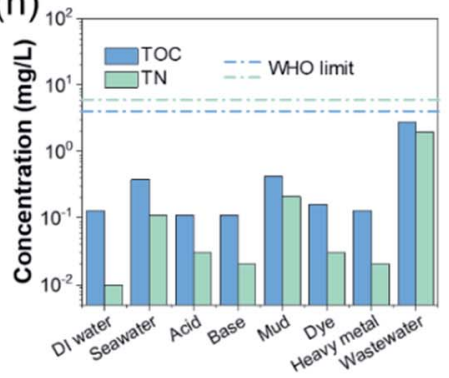

(c)

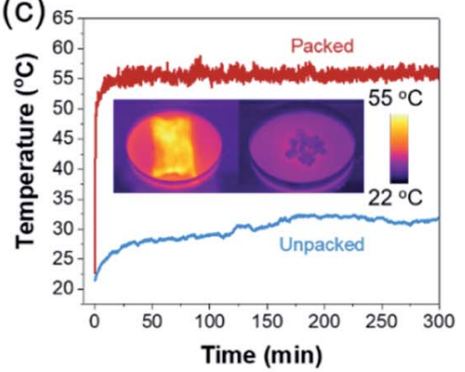

(f)

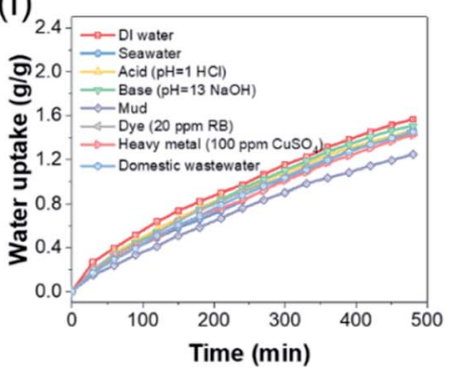

(i)

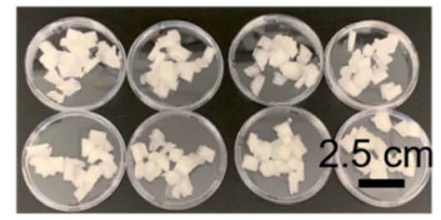

Fig. 4 (a) DVS curve of PAM- $\mathrm{CaCl}_{2}$ hydrogel at $25^{\circ} \mathrm{C}$. (b) Water uptake, (c) photothermal properties, and (d) photothermal-assisted water desorption from the packed SWEAPS and unpacked PAM- $\mathrm{CaCl}_{2}$ hydrogel. (e) Digital photos of SWEAPS on the surface of different water sources. (f) Water uptake of SWEAPS from different water sources. (g) ICP-OES and (h) TOC/TN results of the harvested water from different water sources. (i) Digital photos of the PAM- $\mathrm{CaCl}_{2}$ hydrogel after the water sorption and desorption test.

the test of AWH material was also increased stepwise, which was aligned with the changing of $\mathrm{RH}$.

Then the water vapor sorption properties of SWEAPS (denoted as packed) were investigated and compared with those of the $\mathrm{AWH}$ material (i.e., $\mathrm{PAM}-\mathrm{CaCl}_{2}$ hydrogel, denoted as unpacked) (Fig. 4b and $\mathrm{S} 7 \dagger$ ). As shown in Fig. 4b, the unpacked PAM-CaCl 2 hydrogel shows a slightly higher water vapor sorption rate than that of the packed SWEAPS. The weight change difference between unpacked and packed at $t=240,480$, and $720 \mathrm{~min}$ was $0.17,0.17$, and $0.10 \mathrm{~g} \mathrm{~g}^{-1}$, respectively. The water sorption of SWEAPS was mainly contributed by the encapsulated $\mathrm{PAM}-\mathrm{CaCl}_{2}$ hydrogel, with the contribution of the omniphobic F-AgNPs@SiO ${ }_{2}$ (acotton fabric being negligible (Fig. S8a $\dagger$ ). Owing to the existence of the extra fabric diffusion layer in the packed SWEAPS, the water vapor sorption rate of the packed was lower than that of the unpacked. However, at the end of the test $(t=1000 \mathrm{~min})$, the weight change difference of the packed and unpacked was close to zero. This result further confirms that the omniphobic layer does not influence the water sorption properties of the AWH material.

The photothermal properties of the materials were investigated under simulated solar irradiation with a light intensity of
$1000 \mathrm{~W} \mathrm{~m}^{-2}$ (1 sun). As shown in Fig. 4c, the temperature of the unpacked $\mathrm{PAM}-\mathrm{CaCl}_{2}$ hydrogel changed slowly and remained below $30{ }^{\circ} \mathrm{C}$ during most of the test, while the packed was quickly heated up from $22{ }^{\circ} \mathrm{C}$ to $\sim 55{ }^{\circ} \mathrm{C}$ within $10 \mathrm{~min}$. This is because $\sim 80 \%$ of the incident solar light was absorbed and converted to heat by the F-AgNPs@SiO ${ }_{2} @$ cotton fabric (Fig. S8b $\dagger$ ) largely due to the localized surface plasmon resonance effect of AgNPs. Only $\sim 10 \%$ of the sorbed water was released from the unpacked $\mathrm{PAM}-\mathrm{CaCl}_{2}$ hydrogel under the 1 sun irradiation at the end of $300 \mathrm{~min}$. In contrast, $\sim 80 \%$ of the sorbed water was released from SWEAPS (Fig. 4d) under otherwise the same conditions. As can be seen, although the photothermal performance of the F-AgNPs@SiO ${ }_{2}$ @cotton fabric may not be competitive with conventional photothermal materials, such as carbon nanotubes, graphene/graphene oxide, and MXenes, it can still be used to satisfactorily assist the release of the sorbed water under solar irradiation.

\section{Water uptake from different water sources}

To simulate potentially complicated and various application situations, 8 batches of bulk source water with different contaminants, including (1) DI water as the reference, (2) 
seawater, (3) acidic aqueous solution with $\mathrm{pH}=1$, (4) alkaline aqueous solution with $\mathrm{pH}=13$, (5) mud, (6) rhodamine B dye solution, (7) $\mathrm{CuSO}_{4}$ aqueous solution, and (8) untreated domestic wastewater (pre-filtered to remove solid components), were tested to evaluate the clean water production capability of SWEAPS. Before the water sorption experiments, we carefully measured the relative humidity right above the bulk water surface. The humidity sensor was placed $\sim 0.5 \mathrm{~cm}$ above the water surface and the measured $\mathrm{RH}$ ranged between 97 and 99\% (note: 99\% RH was the maximal reading the humidity meter can produce) for all these source waters except the mud whose RH was measured to be $\sim 89 \%$ at $\sim 0.5 \mathrm{~cm}$ above its surface. Our measurements convincingly confirm very stably high RH at the surface of bulk water.

In doing water uptake experiments, SWEAPS with $2.0 \mathrm{~g}$ of the AWH sorbent was put and floated on the surface of these water sources (Fig. 4e). Due to the high RH (97-99\%) right above the bulk water (water samples 1-4 and 6-8, Fig. 4e), SWEAPS showed high water uptake ( $>1.43 \mathrm{~g} \mathrm{~g}^{-1}$ in $8 \mathrm{~h}$, Fig. $\left.4 \mathrm{f}\right)$. Among them, SWEAPS with DI water had the highest water uptake capacity of $\sim 1.57 \mathrm{~g} \mathrm{~g}^{-1}$, twice its water uptake capacity from the ambient air of $\sim 55-60 \% \mathrm{RH}\left(\sim 0.81 \mathrm{~g} \mathrm{~g}^{-1}\right.$, Fig. $\left.4 \mathrm{~b}\right)$. SWEAPS with the mud had the lowest water uptake $\left(\sim 1.25 \mathrm{~g} \mathrm{~g}^{-1}\right)$, presumably due to the relatively low $\mathrm{RH}(\sim 89 \%)$ above the surface of the mud. Please be noted that these water uptake capacities are not the saturation capacities of SWEAPS. For instance, extending the water sorption time further to $72 \mathrm{~h}$, the water uptake of SWEAPS from the DI water surface can be increased to $\sim 2.38 \mathrm{~g}$ $\mathrm{g}^{-1}$. The stability of SWEAPS was further investigated by conducting 10 cycles of water uptake from bulk DI water (8 hours per cycle) and water release under simulated sunlight (1 kW $\mathrm{m}^{-2}, 3$ hours per cycle). The contact angle of water at the omniphobic fabric before and after the cycling test was also measured. The results have been listed in Fig. S9.† As can be seen, neither water uptake capacity nor photothermal water release performance displays significant differences. The water contact angle of the omniphobic encapsulation layer before and after the cycling test remains almost identical (i.e., $151^{\circ}$ before the test $v s .148^{\circ}$ after the test). These results demonstrate the recyclability of the device.

In order to collect the sorbed water, the desorption process of water-sorbed SWEAPS was conducted in a closed container with a metal chamber as the collector and a transparent dish as the cover. A spectrally selective absorber (SSA) layer was used as the photothermal component to assist the water evaporation. SWEAPS was covered by the SSA layer on the top and then placed inside the metal container (Fig. S10 $\dagger$ ). Due to the light reflection of the metal chamber wall, the bottom side of SWEAPS can receive the reflected light to enable its own photothermal process, which further facilitates water evaporation (Fig. S10a $\dagger$ ). After $3 \mathrm{~h}$ irradiation with $1 \mathrm{~kW} \mathrm{~m}{ }^{-2}$ sunlight, $\sim 1 \mathrm{~mL}$ of water was collected from the collector. The same experiment was performed 3 more times to collect enough water sample for water quality analysis.

The quality of the collected water from 8 batches was further examined by ICP-OES and TOC/TN analysis to evaluate the metal ions and organic compound concentration (Fig. $4 \mathrm{~g}$ and h). As can be seen, all 8 collected water samples met the WHO standards for these individual measured parameters. ${ }^{36}$ Importantly, although the TOC and TN concentrations in the collected water from the domestic wastewater batch are significantly higher than the rest, they are still below the WHO limits. In a typical photothermal distillation process, source water is in situ heated to enhance the water evaporation, and the high local temperature in turn unproductively promotes VOCs' volatilization, leading to the VOC enrichment in the produced water. ${ }^{\mathbf{8}, 37}$ Fortunately, SWEAPS collects water vapor at a lower temperature (i.e., ambient temperature) and, also due to the high selectivity of the vapor sorbent towards water vapor, the VOC enrichment issue is minimized in the process of extracting water vapor from the contaminated water source by SWEAPS, which is convincingly corroborated by the satisfactorily low TOC and TN concentrations of the collected water from the untreated domestic wastewater by SWEAPS. In addition, all the hydrogel sorbents after the water sorption-desorption tests retain their original white color without being tinted by the contaminated water sources (Fig. 4i), further indicating the excellent pollutant shielding effect of the omniphobic fabric. These results suggest the feasibility of producing clean water by SWEAPS from various contaminated bulk water sources.

\section{Water production from air and seawater under real outdoor conditions}

To demonstrate the practical clean water production performance of SWEAPS under real outdoor conditions, the water uptake-water collection tests were conducted in the Red Sea of Saudi Arabia. In the water uptake step, SWEAPS with $15 \mathrm{~g}$ of the pre-dried PAM-CaCl ${ }_{2}$ hydrogel was floated on the surface of the Red Sea inside the KAUST campus from 16:00 to 24:00 on Dec. 17, 2020 (Fig. 5a). The temperature above the Red Sea during the water uptake process was $\sim 20^{\circ} \mathrm{C}$. Due to the lack of the tools to measure the weight change of SWEAPS on the Red Sea surface in real time, only the weight of SWEAPS after the water uptake step was measured and recorded. After $8 \mathrm{~h}$ water sorption, $\sim 13 \mathrm{~g}$ of water was sorbed by SWEAPS. In order to evaluate the potential of SWEAPS to produce water at night, the water collection process was conducted with battery-powered electric heating plates for $1.5 \mathrm{~h}$ (1:00-2:30 am, Dec. 18, 2020). The detailed configuration of the water collection system is shown in Fig. $5 \mathrm{~b}$ and c. In more details, SWEAPS was tightly bound to the electric heating plate and sealed in a zipper bag. The battery was equipped with an inverter to power up the electric heating plate and to maintain the heater temperature at $70{ }^{\circ} \mathrm{C}$. The bottom of the heating plate was covered with a sponge tape to prevent heat loss from the bottom. A total of $\sim 9.5 \mathrm{~g}$ of clean water was collected inside the zipper bag (Fig. 5d-f). Since the battery can be charged by a PV panel during the daytime, the electricity-powered-heating approach allows the system to be able to produce clean water during the night in the absence of sunlight.

In comparison, clean water production from air was also performed by the same SWEAPS. SWEAPS with $15 \mathrm{~g}$ of pre-dried PAM- $\mathrm{CaCl}_{2}$ hydrogel was kept outdoor from 18:00 pm, Nov. 17, 
Dec. 17,2020 16:00-24:00
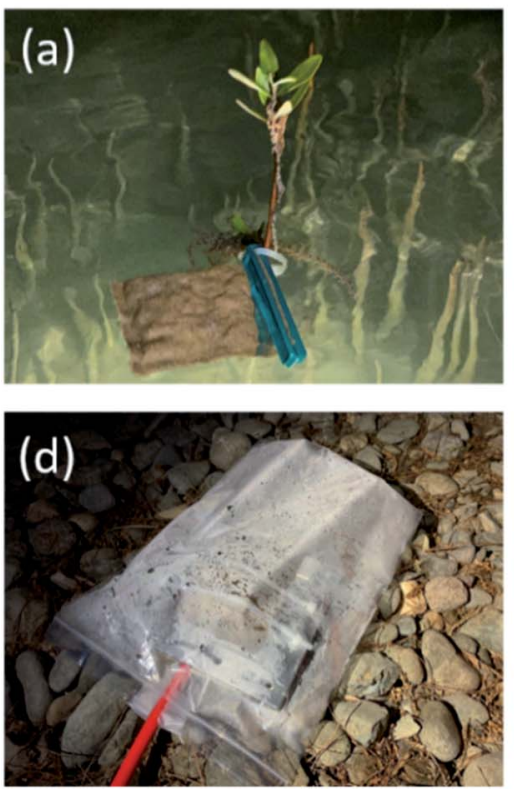

Dec. 18,2020 01:00-02:30
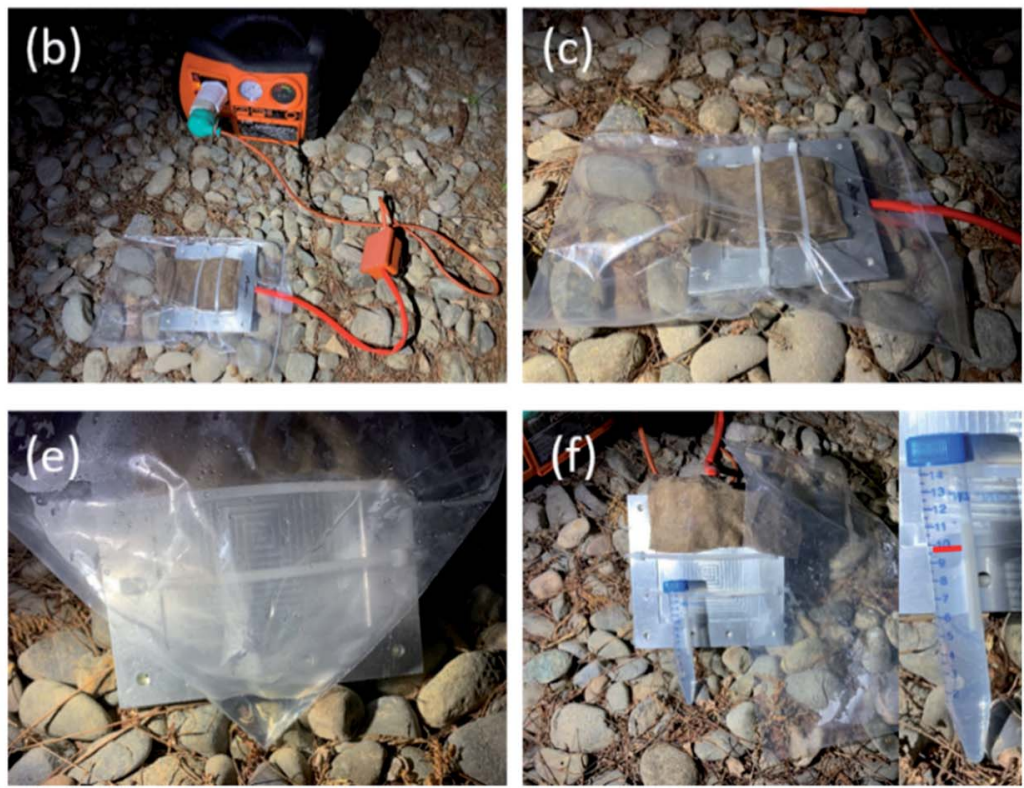

Fig. 5 (a) Digital photo of SWEAPS fixed on a mangrove branch in the Red Sea inside the KAUST campus. (b) Experimental setup of the batterypowered water collection system. (c) Detailed configuration of the collection system, which consists of an electric heating plate, a plastic zipper bag, and a saturated hybrid on the electric heating plate. (d) Condensed water droplets on the inner surface of the zipper bag. (e) Close-up of the condensed water. (f) Collected water in a $15 \mathrm{~mL}$ centrifuge tube.

2020 to $6: 00$ am, Nov. 18, $2020(\mathrm{GMT}+3)$ for water sorption directly from the ambient air (Fig. 6a). The temperature and $\mathrm{RH}$ during the test were recorded (Fig. 6b), which ranged between 22 and $28{ }^{\circ} \mathrm{C}$, and $\sim 55$ and $75 \%$, respectively. The wind speed at the testing site was read from the meter and was between 0 and $1.5 \mathrm{~m} \mathrm{~s}^{-1}$ on average. After $12 \mathrm{~h}$ water sorption, $\sim 8.7 \mathrm{~g}$ of water was sorbed by SWEAPS (Fig. 6c).
For the photothermal-assisted water desorption and collection, a SSA layer was used as the photothermal material and SWEAPS was placed on one side of the SSA, a metal box $(20 \times 20$ $\times 5 \mathrm{~cm}$ ) was used as the condensation chamber, and a polypropylene film was used as a transparent window and condensation layer (Fig. 5d). The sunlight-induced water collection was started on Nov. 18, 2020, at 14:30 local time (GMT+3). The (a)

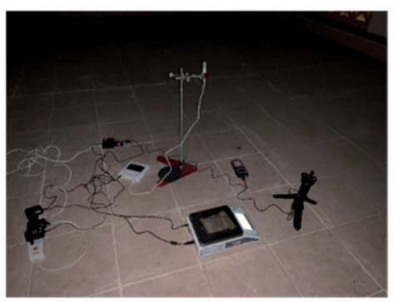

(d)

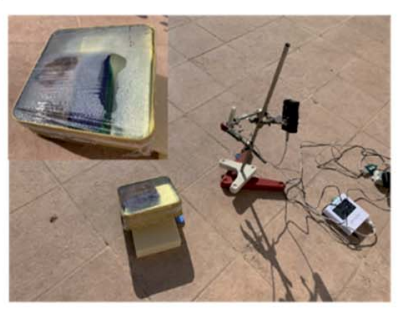

(b)
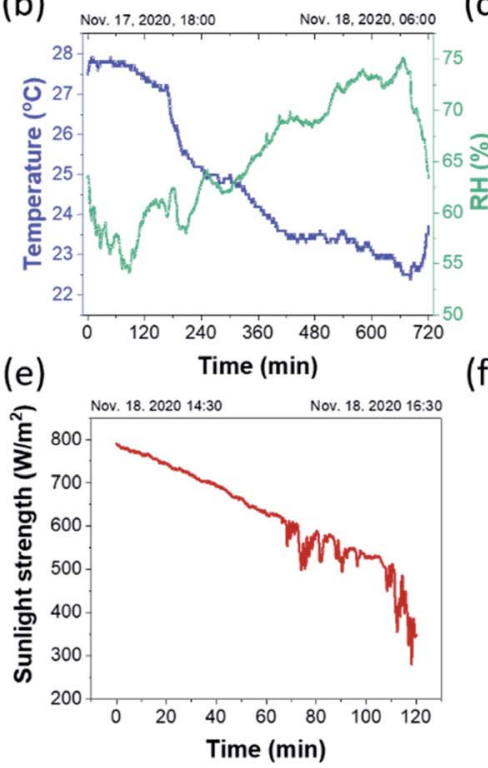

(c)

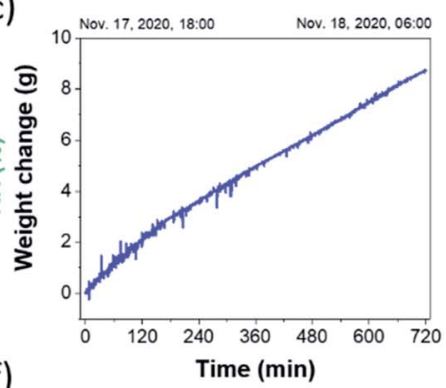

(f)

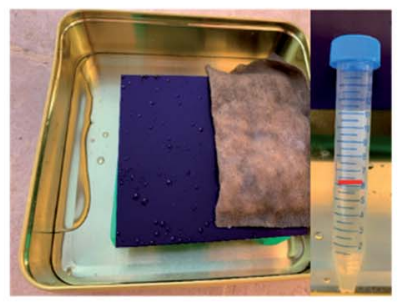

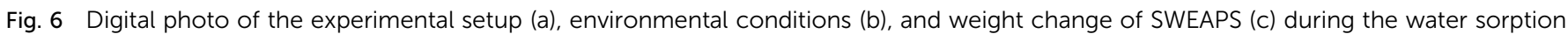

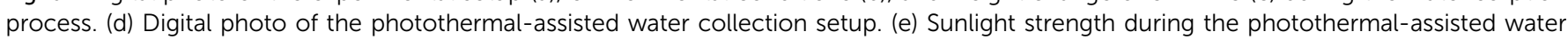
collection test. (f) The condensed water inside the chamber and after being collected in a $15 \mathrm{~mL}$ centrifuge tube. 
assembled AWH device was tilted $\sim 20^{\circ}$ facing the south to gain sufficient solar irradiation (Fig. 5d). Under the solar irradiation, the F-AgNPs@SiO ${ }_{2} @$ cotton fabric absorbs sunlight and converts it into heat to heat the $\mathrm{PAM}-\mathrm{CaCl}_{2}$ hydrogel inside and, meanwhile, the SSA layer serves as a "hot plate" to heat up SWEAPS from the bottom. The back surface of the SSA layer was covered with sponge tape to reduce heat loss at the bottom. Due to the weakened sunlight strength in the afternoon of the winter (Fig. 5e), the water production rate was limited, but SWEAPS still showed good clean water production capacity, with $\sim 6 \mathrm{~g}$ of water collected after $2 \mathrm{~h}$ sunlight irradiation.

The two batches of outdoor tests successfully demonstrate the feasibility of SWEAPS to produce clean water from bulkphase water under field conditions. Also, the photothermalbased and electricity-powered-heating approaches enable SWEAPS to produce clean water continuously around the clock, which can be essential for emergency response.

\section{Conclusion}

This work proposed and demonstrated a SWEAPS design, composed of AWH sorbent encapsulated by an omniphobic fabric, to produce clean water from a wide range of bulk water resources with impaired quality. SWEAPS shows long-term stability and excellent pollution shielding ability which enables it to produce clean water even from real untreated domestic wastewater. Compared to the traditional $\mathrm{AWH}$ approach, SWEAPS shows significantly improved water uptake capacity from liquid water surfaces owing to the high relative humidity there. SWEAPS is promising to meet the basic water demand in places where only polluted water sources are available.

\section{Conflicts of interest}

There are no conflicts to declare.

\section{References}

1 L. Zhang, Z. Xu, L. Zhao, B. Bhatia, Y. Zhong, S. Gong and E. N. Wang, Passive, high-efficiency thermally-localized solar desalination, Energy Environ. Sci., 2021, 14(4), 17711793.

2 P. Wang, Emerging investigator series: the rise of nanoenabled photothermal materials for water evaporation and clean water production by sunlight, Environ. Sci.: Nano, 2018, 5(5), 1078-1089.

3 L. Zhou, X. Li, G. W. Ni, S. Zhu and J. Zhu, The revival of thermal utilization from the Sun: interfacial solar vapor generation, Natl. Sci. Rev., 2019, 6(3), 562-578.

4 M. M. Mekonnen and A. Y. Hoekstra, Four billion people facing severe water scarcity, Sci. Adv., 2016, 2(2), e1500323.

5 World Health Organization (WHO), Drinking Water, 2019, https://www.who.int/news-room/fact-sheets/detail/drinkingwater.

6 E. Lipczynska-Kochany, Effect of climate change on humic substances and associated impacts on the quality of surface water and groundwater: a review, Sci. Total Environ., 2018, 640-641, 1548-1565.

7 L. Andrade, J. O'Dwyer, E. O'Neill and P. Hynds, Surface water flooding, groundwater contamination, and enteric disease in developed countries: a scoping review of connections and consequences, Environ. Pollut., 2018, 236, 540-549.

8 L. Shi, Y. Shi, S. Zhuo, C. Zhang, Y. Aldrees, S. Aleid and P. Wang, Multi-functional 3D honeycomb ceramic plate for clean water production by heterogeneous photo-Fenton reaction and solar-driven water evaporation, Nano Energy, 2019, 60, 222-230.

9 L. Shi, Y. Shi, R. Li, J. Chang, N. Zaouri, E. Ahmed, Y. Jin, C. Zhang, S. Zhuo and P. Wang, SiC-C composite as a highly stable and easily regenerable photothermal material for practical water evaporation, ACS Sustainable Chem. Eng., 2018, 6(7), 8192-8200.

10 F. He, M. Han, J. Zhang, Z. Wang, X. Wu, Y. Zhou, L. Jiang, S. Peng and Y. Li, A simple, mild and versatile method for preparation of photothermal woods toward highly efficient solar steam generation, Nano Energy, 2020, 71, 104650.

11 L. Zhu, L. Sun, H. Zhang, H. Aslan, Y. Sun, Y. Huang, F. Rosei and M. Yu, A solution to break the salt barrier for high-rate sustainable solar desalination, Energy Environ. Sci., 2021, 14(4), 2451-2459.

12 X. Wu, M. E. Robson, J. L. Phelps, J. S. Tan, B. Shao, G. Owens and $\mathrm{H}$. $\mathrm{Xu}$, A flexible photothermal cotton-CuS nanocageagarose aerogel towards portable solar steam generation, Nano Energy, 2019, 56, 708-715.

13 Y. Tu, R. Wang, Y. Zhang and J. Wang, Progress and Expectation of Atmospheric Water Harvesting, Joule, 2018, 2(8), 1452-1475.

14 X. Zhou, H. Lu, F. Zhao and G. Yu, Atmospheric Water Harvesting: A Review of Material and Structural Designs, ACS Mater. Lett., 2020, 2(7), 671-684.

15 A. LaPotin, H. Kim, S. R. Rao and E. N. Wang, Adsorptionbased atmospheric water harvesting: impact of material and component properties on system-level performance, Acc. Chem. Res., 2019, 52(6), 1588-1597.

16 R. Li, Y. Shi, M. Wu, S. Hong and P. Wang, Photovoltaic panel cooling by atmospheric water sorption-evaporation cycle, Nat. Sustain., 2020, 3(8), 636-643.

17 H. Kim, S. Yang, S. R. Rao, S. Narayanan, E. A. Kapustin, H. Furukawa, A. S. Umans, O. M. Yaghi and E. N. Wang, Water harvesting from air with metal-organic frameworks powered by natural sunlight, Science, 2017, 356(6336), 430434.

18 C. Wang, L. Hua, H. Yan, B. Li, Y. Tu and R. Wang, A thermal management strategy for electronic devices based on moisture sorption-desorption processes, Joule, 2020, 4(2), 435-447.

19 A. Mulchandani, S. Malinda, J. Edberg and P. Westerhoff, Sunlight-driven atmospheric water capture capacity is enhanced by nano-enabled photothermal desiccants, Environ. Sci.: Nano, 2020, 7(9), 2584-2594.

20 R. Li, Y. Shi, M. Alsaedi, M. Wu, L. Shi and P. Wang, Hybrid hydrogel with high water vapor harvesting capacity for 
deployable solar-driven atmospheric water generator, Environ. Sci. Technol., 2018, 52(19), 11367-11377.

21 R. Li, Y. Shi, M. Wu, S. Hong and P. Wang, Improving atmospheric water production yield: enabling multiple water harvesting cycles with nano sorbent, Nano Energy, 2020, 67, 104255.

22 H. Qi, T. Wei, W. Zhao, B. Zhu, G. Liu, P. Wang, Z. Lin, X. Wang, X. Li, X. Zhang and J. Zhu, An interfacial solardriven atmospheric water generator based on a liquid sorbent with simultaneous adsorption-desorption, $A d v$. Mater., 2019, 31(43), 1903378.

23 A. LaPotin, Y. Zhong, L. Zhang, L. Zhao, A. Leroy, H. Kim, S. R. Rao and E. N. Wang, Dual-stage atmospheric water harvesting device for scalable solar-driven water production, Joule, 2021, 5(1), 166-182.

24 J. Xu, T. Li, J. Chao, S. Wu, T. Yan, W. Li, B. Cao and R. Wang, Efficient solar-driven water harvesting from arid air with metal-organic frameworks modified by hygroscopic salt, Angew. Chem., Int. Ed., 2020, 59(13), 5202-5210.

25 A. Mulchandani and P. Westerhoff, Geospatial climatic factors influence water production of solar desiccant driven atmospheric water capture devices, Environ. Sci. Technol., 2020, 54(13), 8310-8322.

26 B. Gido, E. Friedler and D. M. Broday, Assessment of atmospheric moisture harvesting by direct cooling, Atmos. Res., 2016, 182, 156-162.

27 B. Gido, E. Friedler and D. M. Broday, Liquid-desiccant vapor separation reduces the energy requirements of atmospheric moisture harvesting, Environ. Sci. Technol., 2016, 50(15), 8362-8367.

28 D. K. Nandakumar, Y. Zhang, S. K. Ravi, N. Guo, C. Zhang and S. C. Tan, Solar energy triggered clean water harvesting from humid air existing above sea surface enabled by a hydrogel with ultrahigh hygroscopicity, Adv. Mater., 2019, 31(10), 1806730.
29 M. Wu, B. Ma, T. Pan, S. Chen and J. Sun, Silvernanoparticle-colored cotton fabrics with tunable colors and durable antibacterial and self-healing superhydrophobic properties, Adv. Funct. Mater., 2016, 26(4), 569-576.

30 B. Leng, Z. Shao, G. de With and W. Ming, Superoleophobic cotton textiles, Langmuir, 2009, 25(4), 2456-2460.

31 G. Franci, A. Falanga, S. Galdiero, L. Palomba, M. Rai, G. Morelli and M. Galdiero, Silver nanoparticles as potential antibacterial agents, Molecules, 2015, 20(5), 88568874.

32 S. Shrivastava, T. Bera, A. Roy, G. Singh, P. Ramachandrarao and D. Dash, Characterization of enhanced antibacterial effects of novel silver nanoparticles, Nanotechnology, 2007, 18(22), 225103.

33 W.-R. Li, X.-B. Xie, Q.-S. Shi, H.-Y. Zeng, Y.-S. Ou-Yang and Y.-B. Chen, Antibacterial activity and mechanism of silver nanoparticles on Escherichia coli, Appl. Microbiol. Biotechnol., 2010, 85(4), 1115-1122.

34 R. Li, Y. Shi, M. Altunkaya, S. Aleid, C. Zhang, W. Wang and P. Wang, Metal- and halide-free, solid-state polymeric water vapor sorbents for efficient water-sorption-driven cooling and atmospheric water harvesting, Mater. Horiz., 2021, 8, 1518-1527.

35 G. S. Frankel, J. D. Vienna, J. Lian, J. R. Scully, S. Gin, J. V. Ryan, J. Wang, S. H. Kim, W. Windl and J. Du, A comparative review of the aqueous corrosion of glasses, crystalline ceramics, and metals, npj Mater. Degrad., 2018, 2(1), 15.

36 World Health Organization (WHO), Guidelines for DrinkingWater Quality, 4th edn, incorporating the 1st addendum, https:/www.who.int/publications/i/item/9789241549950, 2017.

37 R. Chen, T. Zhang, J. Kim, H. Peng, M. Ye and C.-H. Huang, Interfacial solar distillation for freshwater production: fate of volatile and semivolatile organic contaminants, Environ. Sci. Technol., 2021, 55(9), 6248-6256. 